\title{
Características bio-ópticas y morfometría de una laguna de zona templada
}

\section{Bio-optical characteristics and morphometry of a temperate zone lake}

\author{
Vanesa Yael Bohn*, María Cintia Piccolo** \\ y Gerardo Miguel Eduardo Perillo ***
}

\section{INTRODUCCIÓN}

Las superficies acuáticas absorben o transmiten la mayor parte de la radiación óptica que reciben, siendo mayor su capacidad de absorción cuanto mayor sea la longitud de onda (Pinilla, 1995). De esta manera, la curva espectral del agua muestra una reducción en los valores de reflectancia con el incremento de la longitud de onda. Como consecuencia, en aguas claras y profundas la reflectancia se aproxima a cero en el rango del infrarrojo cercano (Mather, 2004).

La erosión del suelo es la mayor causa de contaminación de aguas superficiales. La degradación de la calidad del agua superficial es causada por sedimentación, contaminantes y nutrientes (Han, 1997). La calidad del agua también está afectada por la biomasa fitoplanctónica (clorofila «a») y otras partículas de origen orgánico. Estos componentes son, asimismo, los princi-

\footnotetext{
* Departamento de Geografía y Turismo, Universidad Nacional del Sur (UNS) (vbohn@ criba.edu.ar).

${ }^{* *}$ Departamento de Geografía y Turismo, Universidad Nacional del Sur (UNS) y CONICET Instituto Argentino de Oceanografía (IADO-CONICET).

*:** Departamento de Geología, Universidad Nacional del Sur (UNS) e Instituto Argentino de Oceanografía (IADO-CONICET).
} 
pales factores que controlan las características espectrales en los cuerpos de agua (Hudson et al., 1994).

Diversos estudios han demostrado la posibilidad de emplear sensores remotos para cartografiar el contenido y la distribución espacial de clorofila «a» en agua (Pinilla, 1995; Rodríguez et al., 2007), grados de eutrofización (Rodríguez et al., 2007; Canziani et al., 2008) y sólidos en suspensión (Ferratti et al., 2008). Thiemann y Kaufmann (2000) demostraron con éxito el empleo de datos satelitales y espectros de reflectancia en la determinación del estado trófico y concentraciones de clorofila «a» en cinco lagos alemanes. A escala local, Canziani et al. (2008) utilizaron datos satelitales y espectrales en complemento con redes neuronales para la estimación de clorofila «a» y sedimentos en suspensión en lagos pampeanos argentinos. Este hallazgo permitió modelar la variación temporal de ambas variables en lagunas poco profundas y turbias.

El objetivo de este trabajo fue evaluar la aplicación de sensores de campo y satelitales en la caracterización bio-óptica de la laguna Chasicó, una laguna profunda de zona templada. Los parámetros morfométricos de la laguna se obtuvieron a partir de datos in situ (por ejemplo, profundidad) y procesamiento digital de imágenes satelitales (por ejemplo, perímetro, área, longitud máxima total).

ÁREA DE ESTUDiO

La laguna Chasicó se localiza en el sudoeste de la provincia de Buenos Aires (Argentina), en el área deprimida de la vertiente suroeste del sistema serrano de Ventania. Es un sistema cerrado que recibe las aguas del arroyo homónimo y de sus afluentes (figura 1). El drenaje de las sierras se encauza a través de un conjunto de pequeños cursos que desaguan en el arroyo Chasicó. $\mathrm{Su}$ sistema de recarga se produce por la aportación directa de las precipitaciones, a través del proceso de infiltración de las aguas subterráneas y por el aporte de los escurrimientos superficiales de una amplia cuenca hidrográfica de $3.764 \mathrm{~km}^{2}$ (Bonorino, 1991) que descarga y encuentra su nivel de base sobre la depresión lagunar que se extiende sobre la cota de -20 msnm (figura 1). En la actualidad su desarrollo espacial es de aproximadamente $50 \mathrm{~km}^{2}$.

El clima de la región en la que se localiza la laguna es templado, con veranos e inviernos bien definidos, y primaveras y otoños moderados. Los valores mensuales de temperatura oscilan entre los 14 y los $20^{\circ} \mathrm{C}$. En general, las precipitaciones presentan dos máximos en primavera y otoño (específicamente 


\section{FIGURA 1}

LOCALIZACIÓN DE LA LAGUNA CHASICÓ (a) EN LA PROVINCIA

DE BUENOS AIRES, ARGENTINA. CROQUIS DE LA LOCALIZACIÓN DE LAS ESTACIONES DE MEDICIÓN EN LA LAGUNA CHASICÓ (b)

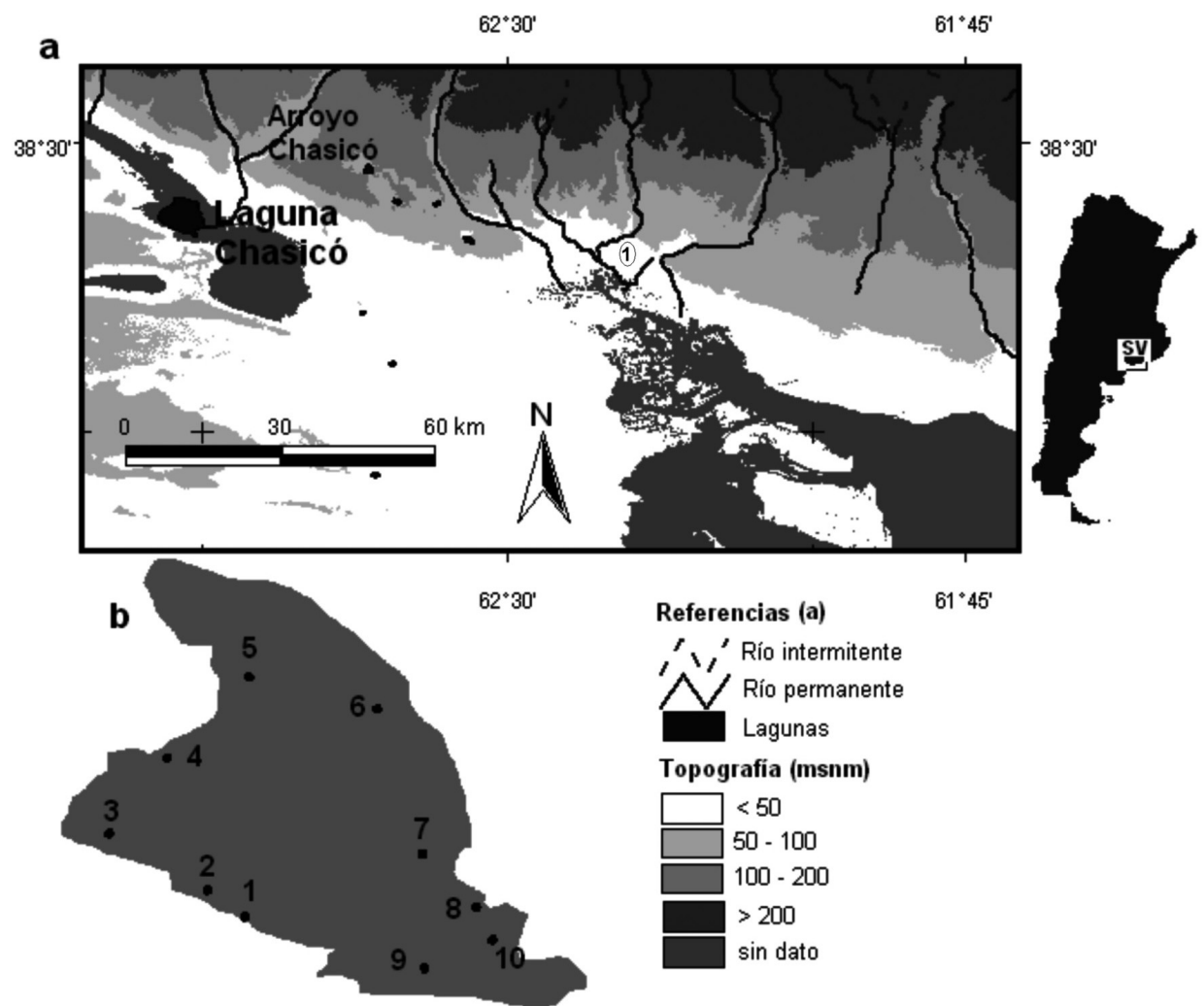

Nota: las estaciones 8 y 10 corresponden al área de la desembocadura del arroyo Chasicó; SV: Sistema de Ventania. Datos topográficos DEM SRTM (USGS); (1): Bahía Blanca.

Fuente: elaboración propia.

en los meses de octubre y mayo). En la ciudad de Bahía Blanca, al SE de la laguna Chasicó (65 km aproximadamente) (figura 1), los vientos presentan una dirección predominante del cuadrante norte y noroeste, durante todas las estaciones del año y una velocidad media anual de $22,5 \mathrm{~km} / \mathrm{h}$ (Campo et al., 2004). En cuanto a los balances hídricos normales, Bahía Blanca presenta un déficit durante los meses de enero-marzo y diciembre mientras que, durante 
los meses de marzo-octubre/noviembre presenta períodos de almacenamiento y recarga (Campo et al., 2004; Bohn, 2009). Las precipitaciones alcanzan los $600 \mathrm{~mm}$ anuales (Ferrelli et al., 2012), disminuyendo hacia el sector de la laguna.

Caracteriza a esta región, la gran variabilidad climática, principalmente las precipitaciones, temperaturas, vientos y humedad relativa ambiente. Las lluvias pueden llegar a valores extremos de bajas precipitaciones, característica común a ambientes áridos o semiáridos, a valores muy por encima del promedio anual similares a regiones extremadamente húmedas (Glave, 2006).

\section{MÉTODO DE TRABAJO}

Para la obtención de parámetros in situ en la laguna Chasicó se utilizaron 10 estaciones de medición, localizadas mediante un Sistema de Posicionamiento Global (GPS) y distribuidas de manera heterogénea (figura 1). Las mediciones se realizaron los días 5 de febrero, 2 de marzo y 12 de abril de 2010. Los valores correspondientes a $\mathrm{pH}$, conductividad eléctrica $(\mathrm{CE}, \mathrm{mS} / \mathrm{cm})$, oxígeno disuelto $(\mathrm{mg} / \mathrm{l})$ y temperatura del agua $\left({ }^{\circ} \mathrm{C}\right)$ fueron obtenidos a nivel superficial mediante el uso de una sonda multiparamétrica Horiba U-10. La transparencia del agua fue estimada mediante la lectura de un Disco Secchi (DS). La profundidad ( $z$ ) se obtuvo mediante una escala graduada. Se tomaron muestras de agua a nivel superficial $(20 \mathrm{~cm})$ para la determinación de concentraciones de clorofila «a» $(\mu \mathrm{g} / \mathrm{l})$. Las mismas fueron analizadas en laboratorio de acuerdo al método espectrofotométrico APHA (1998).

Los valores de reflectancia (R, \%) de la superficie del agua de la laguna fueron obtenidos en campo y también a partir de datos satelitales con el objetivo de caracterizar la laguna desde un punto de vista óptico y desarrollar un modelo de regresión para el cálculo de clorofila «a», respectivamente. En el primer caso, la $\mathrm{R}$ fue medida con un espectroradiómetro de campo manual Ocean Optics Inc ${ }^{a}$ USB2000 (Rango espectral 300-1100 nm) en cada estación de medición (figura 1.b) y para cada fecha. La obtención de los espectros se realizó paralelamente a las mediciones de los parámetros físicos y la recolección de muestras de agua, entre las 10:00 y las 14:00 horas y en condiciones de escasa nubosidad. La calibración de cada una de las mediciones de $\mathrm{R}$ fue realizada usando superficies de reflectancia máxima (blanco) y mínima (negro). La máxima reflectancia se determinó mediante el empleo de una superficie estándar de reflectancia difusa (Ocean Optics WS-1) y la mínima reflectancia se obtuvo mediante el cierre de la entrada de luz a la fibra óptica. 
Las mediciones del mencionado parámetro fueron realizadas en dos ocasiones en forma secuencial y posteriormente promediadas para cada punto de medición (10 estaciones en total). El sensor se colocó a una distancia de $0,50 \mathrm{~m}$ de la superficie. Los espectros fueron obtenidos mediante la conexión del espectroradiómetro de campo y el software SpectraSuite (Ocean Optics, 20012007).

En cuanto a la obtención de $\mathrm{R}$ a partir de datos satelitales, se procesaron las imágenes Landsat 7 ETM+ correspondientes al área de estudio (path/row: 227/087) (Resolución espacial $30 \mathrm{~m}$ ) y próximas a las fechas de medición (13 de febrero, 1 de marzo y 2 de abril de 2010) (CONAE). El procesamiento digital incluyó la corrección geométrica y la conversión de los valores de niveles digitales (ND) a valores de reflectancia (corrección atmosférica) mediante la metodología de Song et al. (2001). Este último procedimiento se realizó como requisito de la utilización de imágenes satelitales de diferentes fechas (estudio multitemporal). Se obtuvieron los valores de $\mathrm{R}$ para cada estación de medición (figura 1.b) mediante el uso del software Envi 4.7. Para la elaboración de la cartografía los datos fueron incorporados al Sistema de Información Geográfica Arc View 3.2.

Para el cálculo del Índice de Vegetación de Diferencia Normalizada (NDVI) (Chuvieco Salinero, 2002) se utilizó

$$
N D V I=\frac{R_{B 4}-R_{B 3}}{R_{B 4}+R_{B 3}}
$$

sobre los datos de $\mathrm{R}$ obtenidos de la imagen satelital donde $\mathrm{R}_{\mathrm{B} 4}=\mathrm{R}$ en la banda 4 de Landsat 7 ETM+ y $\mathrm{R}_{\mathrm{B} 3}=\mathrm{R}$ en la banda 3 de Landsat 7 ETM+.

Se aplicaron modelos de regresión simple entre las concentraciones de clorofila «a» $(\mu \mathrm{g} / \mathrm{l})$ obtenidas en laboratorio y los valores de NDVI calculados a partir de datos satelitales para las dos primeras fechas (febrero y abril de 2010). A partir de esta regresión se modeló y validó la distribución espacial de la clorofila «a» en toda la laguna, píxel a píxel para el mes de abril de 2010. Los parámetros morfométricos de la laguna se obtuvieron a partir de clasificación no supervisada de las imágenes satelitales antes mencionadas: área $\left(\mathrm{km}^{2}\right)$, longitud máxima total (LMT) (km), longitud máxima efectiva (LME) $(\mathrm{km})$, ancho máximo (AM) $(\mathrm{km})$ y desarrollo de línea de costa (DLC) (Hutchinson, 1957; Dangavs, 2005). Para este último parámetro se aplicó

$$
\mathrm{DLC}=\mathrm{P} /\left[2 \cdot(\pi \cdot S)^{1 / 2}\right]
$$

donde $\mathrm{P}$ es el perímetro y $\mathrm{S}$ el área del cuerpo de agua considerado. 


\section{RESULTADOS Y DiSCUSIÓN}

\section{Parámetros físico-químicos}

Los valores de $\mathrm{pH}$, temperatura, CE, transparencia del agua, clorofila «a»y OD fueron relativamente estables entre estaciones de medición (tabla 1). En cuanto a la variación temporal de los parámetros medidos y calculados (tabla 1), se observó homogeneidad en los valores de $\mathrm{CE}, \mathrm{pH}$, clorofila «a» y parámetros morfométricos.

La laguna Chasicó mostró valores medios (período febrero-abril 2010) de clorofila «a» típicos de ambientes mesotróficos (Carlson, 1977) aunque la misma ha sido caracterizada también como un ambiente eutrófico con aguas claras, en otros períodos (Kopprio et al., 2010). Como consecuencia, la transparencia del agua alcanzó valores de $1 \mathrm{~m}$, al igual que fue estimada para la mencionada laguna en estudios previos (Remes Lenicov y Berasain, 2004). La concentración salina hallada a nivel superficial fue muy elevada en relación al resto de las lagunas de la llanura pampeana argentina (Sosnovsky y Quirós, 2005; Bohn et al., 2007; Grosman y Sanzano, 2008; Garibotti et al., 2009) por lo que la laguna estudiada fue caracterizada como polihalina (Ringuelet, 1972). El valor medio de $\mathrm{pH}$ la caracterizó como un cuerpo de agua alcalino.

En relación a los parámetros morfométricos, la laguna mostró homogeneidad temporal. Presentó una forma subrectangular elongada, la cual fue determinada a partir del cálculo del desarrollo de línea de costa (DLC). La LME fue similar a la LMT, lo cual indicó la escasa presencia de sinuosidades en su extensión areal. Su desarrollo espacial alcanzó los $52 \mathrm{~km}^{2}$, similar al área hallada en 2001 (Fiorentino y Paoloni, 2001).

Algunas variaciones temporales se detectaron en relación a la temperatura superficial del agua y a la disponibilidad del oxígeno disuelto. La solubilidad de este último en las aguas superficiales de la laguna fue afectada por la temperatura en forma no lineal, aumentando considerablemente al disminuir la temperatura (tabla 1).

\section{Caracterización bio-óptica de la laguna}

Según datos de reflectancia in situ

Del análisis de los espectros de las 10 estaciones de medición fue posible discriminar, para cada fecha, aquellos correspondientes al área afectada por la 
TABLA 1

PARÁMETROS FÍSICO-QUÍMICOS SUPERFICIALES Y MORFOMÉTRICOS DE LA LAGUNA CHASICÓ DURANTE LOS MESES DE FEBRERO, MARZO Y ABRIL DE 2010

\begin{tabular}{|c|c|c|c|c|}
\hline \multirow{2}{*}{ Parámetro } & \multicolumn{3}{|c|}{ Valor medio entre estaciones } & \multirow{2}{*}{$\begin{array}{l}\text { desvío } \\
\text { estándar }\end{array}$} \\
\hline & febrero & marzo & abril & \\
\hline \multirow{2}{*}{ Temperatura superficial $\left({ }^{\circ} \mathrm{C}\right)^{*}$} & 23,51 & 23,20 & 17,87 & \multirow{2}{*}{ $\pm 3,17$} \\
\hline & $( \pm 0,54)$ & $( \pm 1,04)$ & $( \pm 0,24)$ & \\
\hline \multirow{2}{*}{ Conductividad eléctrica $(\mathrm{mS} / \mathrm{cm})$ * } & 38,78 & 40,36 & 40,56 & \multirow{2}{*}{ $\pm 0,98$} \\
\hline & $( \pm 3,44)$ & $( \pm 0,49)$ & $( \pm 0,45)$ & \\
\hline \multirow{2}{*}{$\mathrm{pH}^{*}$} & 8,68 & 8,74 & 8,79 & \multirow{2}{*}{ $\pm 0,06$} \\
\hline & $( \pm 0,03)$ & $( \pm 0,01)$ & $( \pm 0.01)$ & \\
\hline \multirow{2}{*}{ Oxígeno disuelto $(\mathrm{mg} / \mathrm{L})^{*}$} & 5,92 & 5,92 & 8,61 & \multirow{2}{*}{ $\pm 1,55$} \\
\hline & $( \pm 0,19)$ & $( \pm 0,52)$ & $( \pm 0,22)$ & \\
\hline \multirow{2}{*}{ Transparencia (Disco Secchi, m)* } & 0,82 & 0,78 & 1,00 & \multirow{2}{*}{ $\pm 0,12$} \\
\hline & $( \pm 0,26)$ & $( \pm 0,17)$ & $( \pm 0,16)$ & \\
\hline \multirow{2}{*}{ Clorofila «a» $(\mathrm{g} / \mathrm{l}) *$} & 9,67 & 16,16 & 9,73 & \multirow{2}{*}{ $\pm 3,73$} \\
\hline & $( \pm 3,05)$ & $( \pm 2,77)$ & $( \pm 1,91)$ & \\
\hline Profundidad media hallada $(\mathrm{m})^{*}$ & 4,20 & 4,60 & 4,60 & $\pm 0,23$ \\
\hline Área $\left(\mathrm{km}^{2}\right)$ & 52,62 & 52,18 & 52,00 & $\pm 0,32$ \\
\hline Perímetro $(\mathrm{km})$ & 90,72 & 88,80 & 94,86 & $\pm 3,10$ \\
\hline LMT $(\mathrm{km})$ & 12,10 & 12,08 & 12,24 & $\pm 0,09$ \\
\hline LME (km) & 12,47 & 12,69 & 12,74 & $\pm 0,14$ \\
\hline $\mathrm{AM}(\mathrm{km})$ & 8,04 & 8,00 & 8,28 & $\pm 0,15$ \\
\hline DLC & \multicolumn{3}{|c|}{ Subrectangular elongada } & \\
\hline
\end{tabular}

Nota: el valor indicado para los parámetros con * representa el promedio entre estaciones de medición. El desvío estándar se presenta en la columna para la variación temporal y entre paréntesis para la variación espacial (entre estaciones de medición).

Fuente: elaboración propia.

desembocadura del arroyo Chasicó (figura 1) de las estaciones de medición restantes (valor medio para la laguna) (figura 2). Como resultado del análisis de la variación temporal de los espectros medios, no se observaron diferencias entre las firmas espectrales sino que sólo se detectó un decrecimiento en los valores de R durante el otoño. Durante los meses de febrero y marzo, los valores 
de $\mathrm{R}$ en promedio para todo el espectro fueron superiores al 3,4\%. Para el mes de abril, dicho promedio fue de $2,7 \%$ (figura 2). Este último mes presentó la mayor transparencia del período, lo cual podría explicar el fenómeno (tabla 1).

El valor medio de $\mathrm{R}$ para el mes de febrero fue de $4,5 \%$ y los valores máximos superaron el 6\% en el rango de 540-600 nm de longitud de onda y el 3\% en los 640-660 nm y 680-710 nm de longitud de onda. El primero de los picos máximos registrados indicó presencia de clorofila «a» con el consecuente descenso de los valores de $\mathrm{R}$ en el rango 450-495 nm (azul). En los dos picos máximos restantes (figura 2), los ascensos de $\mathrm{R}$ en los rangos citados podrían estar asociados a la presencia de partículas en suspensión de origen orgánico e inorgánico (altamente reflectivas). Sin embargo, no fue posible determinar el aporte de cada clase de partícula (orgánica o inorgánica) al total del valor de R. Este procedimiento ha sido calificado como muy complejo (Mather, 2004; Sváb et al., 2005). Dicha complejidad es mayor ante el aumento de los sedimentos en suspensión y una distribución heterogénea de los mismos (Lindell et al., 1999).

Durante el mes de marzo, el valor medio de R no superó el 3,5\% mientras que los valores máximos fueron cercanos al 5\% en los 540-600 nm de longitud de onda. En abril, el espectro mostró un valor de R media de 2,7\% superando el $4 \%$ en el rango correspondiente a la respuesta de la clorofila «a». De este modo, los espectros promedio para las estaciones de medición, en el período analizado, mostraron similitudes con aquellos obtenidos para aguas con desarrollo de algas (Han, 1997; Jensen, 2000; Chuvieco Salinero, 2002). Los valores de $\mathrm{R}$ fueron afectados por la presencia de partículas en suspensión de origen orgánico e inorgánico (DS $1 \mathrm{~m}$ ) (tabla 1). En particular, el material de origen orgánico (por ejemplo clorofila «a») provocó un descenso de $\mathrm{R}$ en el rango de longitud de onda 450-500 (azul) y un ascenso de la misma en el rango 500-550 $\mathrm{nm}$ (verde) (figura 2).

En cuanto a las estaciones afectadas por la desembocadura del arroyo Chasicó, los valores obtenidos en febrero se diferenciaron de los dos meses restantes. El espectro afectado por el caudal del arroyo en el mes de febrero mostró valores máximos y medios de $\mathrm{R}$ superiores al 13 y al $9 \%$, respectivamente (figura 2). La R aumentó en forma lineal en relación a la longitud de onda, evidenciando la alta concentración de sedimentos en suspensión (lectura de Disco Secchi $<0,5 \mathrm{~m}$ ). En cuanto a las mediciones realizadas en los meses de marzo y abril, las estaciones cercanas a la afluencia del arroyo Chasicó mostraron una firma espectral similar a los espectros de $\mathrm{R}$ en el resto de la laguna (máximos de $\mathrm{R}$ entre los 520-600 nm). Los máximos valores de R fueron de $9 \%$ (marzo) y de 5,6\% (abril) (figura 2 ). 
FIGURA 2

LOS ESPECTROS DE REFLECTANCIA PROMEDIO ENTRE ESTACIONES DE MEDICIÓN DE LA LAGUNA CHASICÓ NO MOSTRARON VARIACIÓN TEMPORAL RELEVANTE

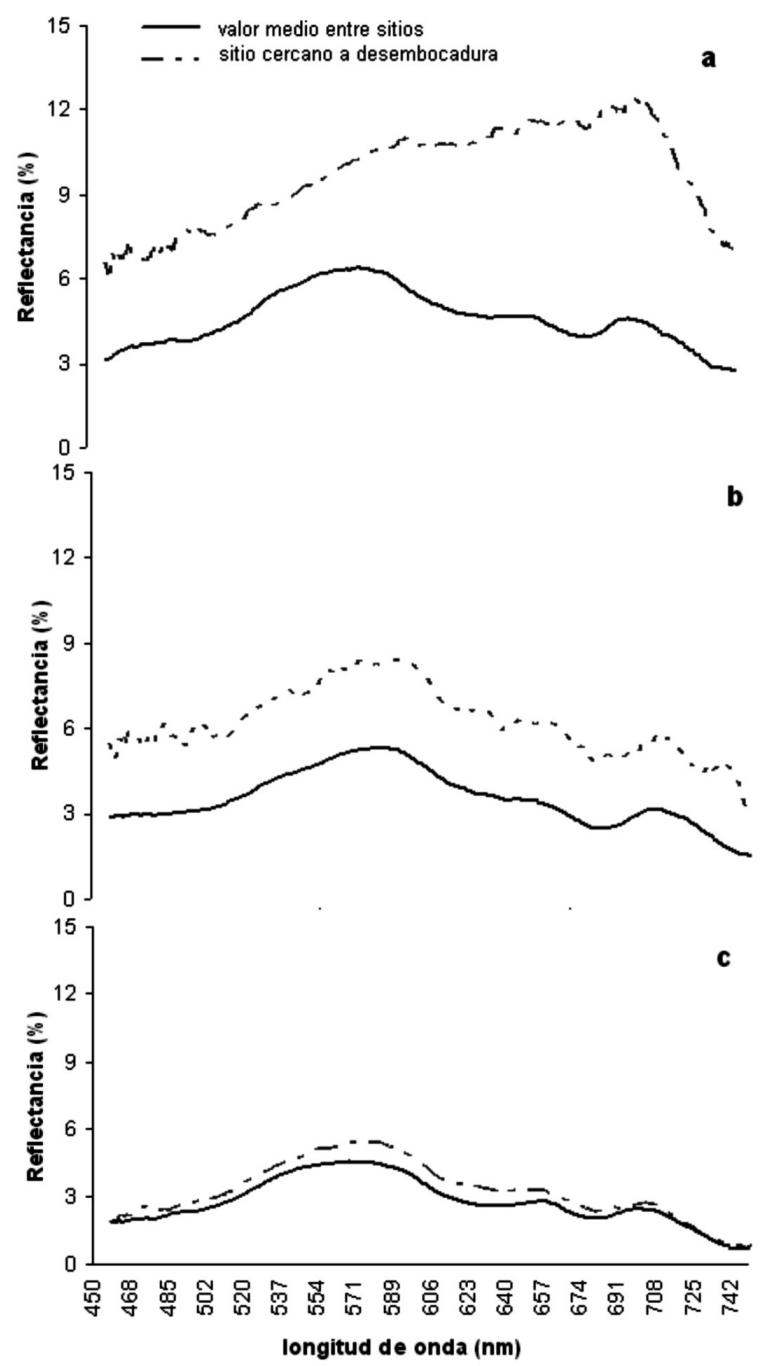

Nota: en las estaciones afectadas por la desembocadura del arroyo homónimo, la reflectancia fue mayor al promedio en todos los casos: a) febrero de 2010, b) marzo de 2010 y c) abril de 2010.

Fuente: elaboración propia. 
En todos los casos, los porcentajes de $\mathrm{R}$ en cercanías de la desembocadura fueron superiores a los valores medios para el resto de la laguna. Ello evidenció el efecto del aporte de sedimentos del arroyo Chasicó y la influencia del intercambio de masas de agua. La diferenciación de este sector de la laguna con respecto a las demás estaciones de medición fue previamente destacada por Bohn et al. (2012) mediante aplicación de métodos estadísticos de agrupamiento sobre datos de reflectividad en un período estival.

Según datos de reflectancia satelitales

Se realizó la transformación de los valores de niveles digitales (ND) a reflectancia (R) de las imágenes satelitales LANDSAT 7 ETM+ (227/087), correspondientes a fechas próximas a la de realización de las mediciones in situ

\section{FIGURA 3}

\section{DISTRIBUCIÓN ESPACIAL DEL NDVI CALCULADO A PARTIR DE DATOS SATELITALES DE REFLECTANCIA (ABRIL 2010)}

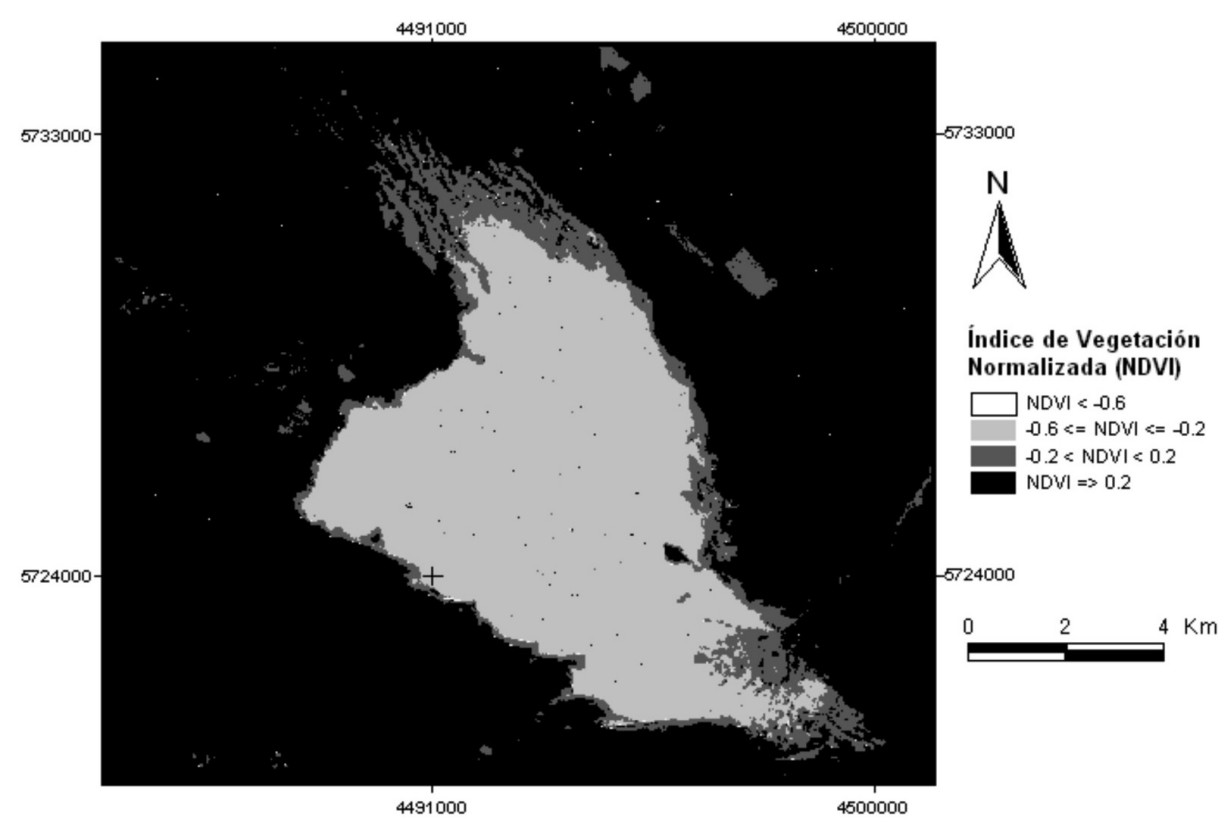

Fuente: elaboración propia.

Estudios Geográficos, Vol. LXXIV, 274, pp. 311-328, enero-junio 2013

ISSN: 0014-1496, eISSN: 1988-8546, doi: 10.3989/estgeogr.201311 
(13 de febrero, 1 de marzo y 2 de abril de 2010). Los valores de $\mathrm{R}$ obtenidos fueron utilizados para el cálculo del NDVI en cada fecha. Los valores de NDVI fueron negativos y cercanos a 0 para el área lagunar, como era de esperar en superficies de agua (Sepulcri et al., 2012). Los mayores valores del índice correspondieron a los sectores cercanos a la costa mientras que el área central de la laguna mostró valores entre - $-0,2$ y -0,6 (figura 3), indicando menor concentración de clorofila. De esta manera, se desarrolló un modelo de regresión simple entre los valores de clorofila «a» medidos in situ y los valores de NDVI de origen satelital correspondientes a los meses de febrero y marzo de 2010. Como resultado, se obtuvo una correlación alta $\left(R^{2}=0,83\right)$ (figura 4$)$.

El modelo de regresión (figura 4) fue aplicado al producto satelital NDVI correspondiente a abril de 2010 para la obtención y cálculo de los valores de clorofila «a» en dicho mes. Como resultado, la correlación entre los valores de clorofila-a in situ y aquellos calculados por el modelo de regresión fue de $\mathrm{R}^{2}=0,96$ (figura 5). Sobre la base de esta regresión simple se modeló la distribución espacial de la clorofila «a» en toda la laguna para el período abril de 2010 (figura 6).

FIGURA 4

MODELO DE REGRESIÓN SIMPLE ENTRE LAS CONCENTRACIONES DE
CLOROFILA «a» IN SITU Y LOS VALORES DE NDVI (AMBOS
CORRESPONDIENTES A FEBRERO Y MARZO DE 2010)

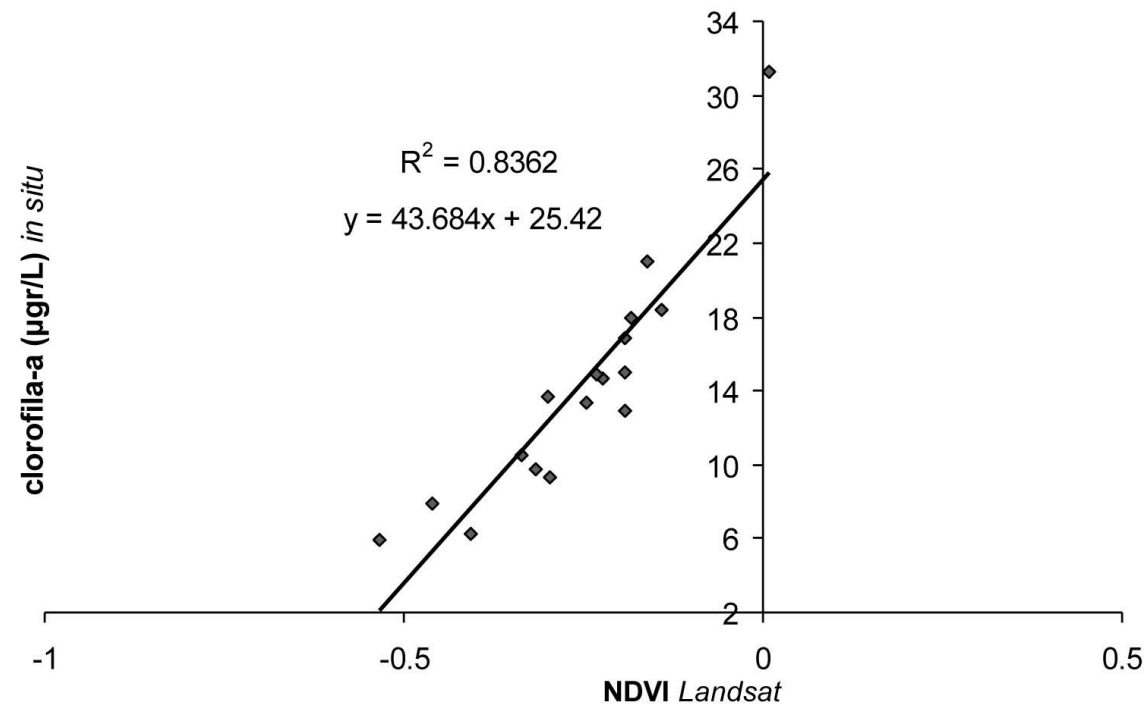

Fuente: elaboración propia. 
FIGURA 5

APLICACIÓN DEL MODELO DE REGRESIÓN PARA EL CÁLCULO DE CONCENTRACIONES DE CLOROFILA «a» EN LA LAGUNA CHASICÓ
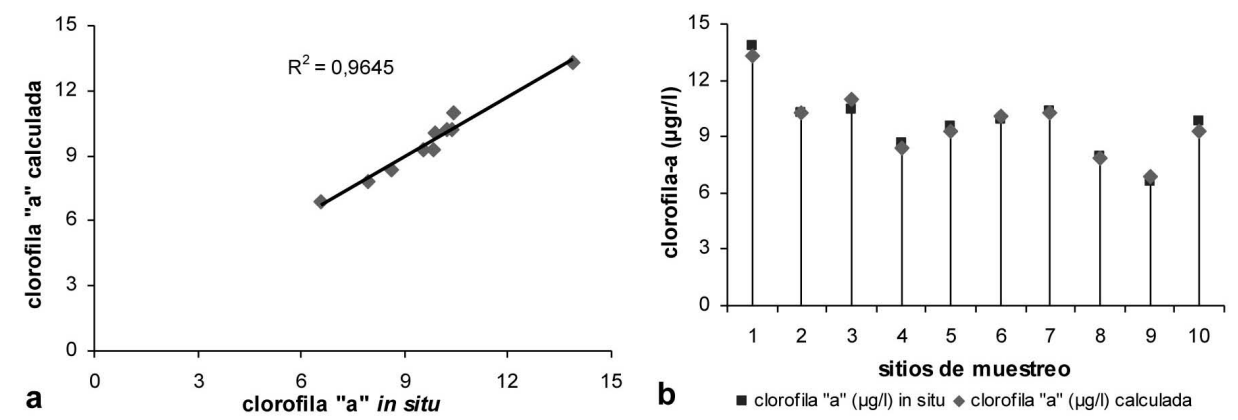

Nota: la aplicación del modelo de regresión para el cálculo de concentraciones de clorofila «a» en la laguna Chasicó mostró una aceptable estimación del feopigmento para el mes de abril de 2010: a) clorofila «a» calculada vs. clorofila «a» in situ (ambas para abril de 2010), b) concentraciones de clorofila «a» medida y calculada según estaciones de medición (abril de 2010).

Fuente: elaboración propia.

Como resultado de la incorporación de los datos al SIG, se obtuvo que las mayores concentraciones de clorofila «a» se localizaron en cercanías a la costa: el 33,8\% del área de la laguna presentó concentraciones de clorofila «a» superiores a $10 \mu \mathrm{g} / \mathrm{l}$. El mayor porcentaje de área de la laguna ( 53\%) mostró concentraciones entre 5 y $10 \mu \mathrm{g} / \mathrm{l}$ mientras que, el 12,8\% restante, mostró concentraciones inferiores a $5 \mu \mathrm{g} / \mathrm{l}$ (figura 6).

\section{CONCLUSIONES}

La utilización de sensores de campo para la medición de $\mathrm{R}$ en la laguna Chasicó permitió diferenciar la influencia de distintas variables en su caracterización bio-óptica. De esta manera, los espectros permitieron observar el predominio de turbidez, menor profundidad o desarrollo de procesos de mezcla tales como la descarga de sedimentos del arroyo Chasicó (sureste de la laguna) en el área de su desembocadura.

Durante el período estudiado, los parámetros morfométricos fueron estables para la laguna. La transparencia del agua también fue relativamente homogénea a escala temporal. Sin embargo, a pesar de que su variabilidad fue escasa, ésta fue detectada mediante el análisis de los espectros de reflectancia. 


\section{FiguRA 6}

DISTRIBUCIÓN ESPACIAL DE LAS CONCENTRACIONES DE CLOROFILA «a» CALCULADA A PARTIR DEL MODELO DE REGRESIÓN (CLOROFILA «a» IN SITU VS. NDVI) SOBRE EL PRODUCTO SATELITAL NDVI CORRESPONDIENTE AL MES DE ABRIL DE 2010

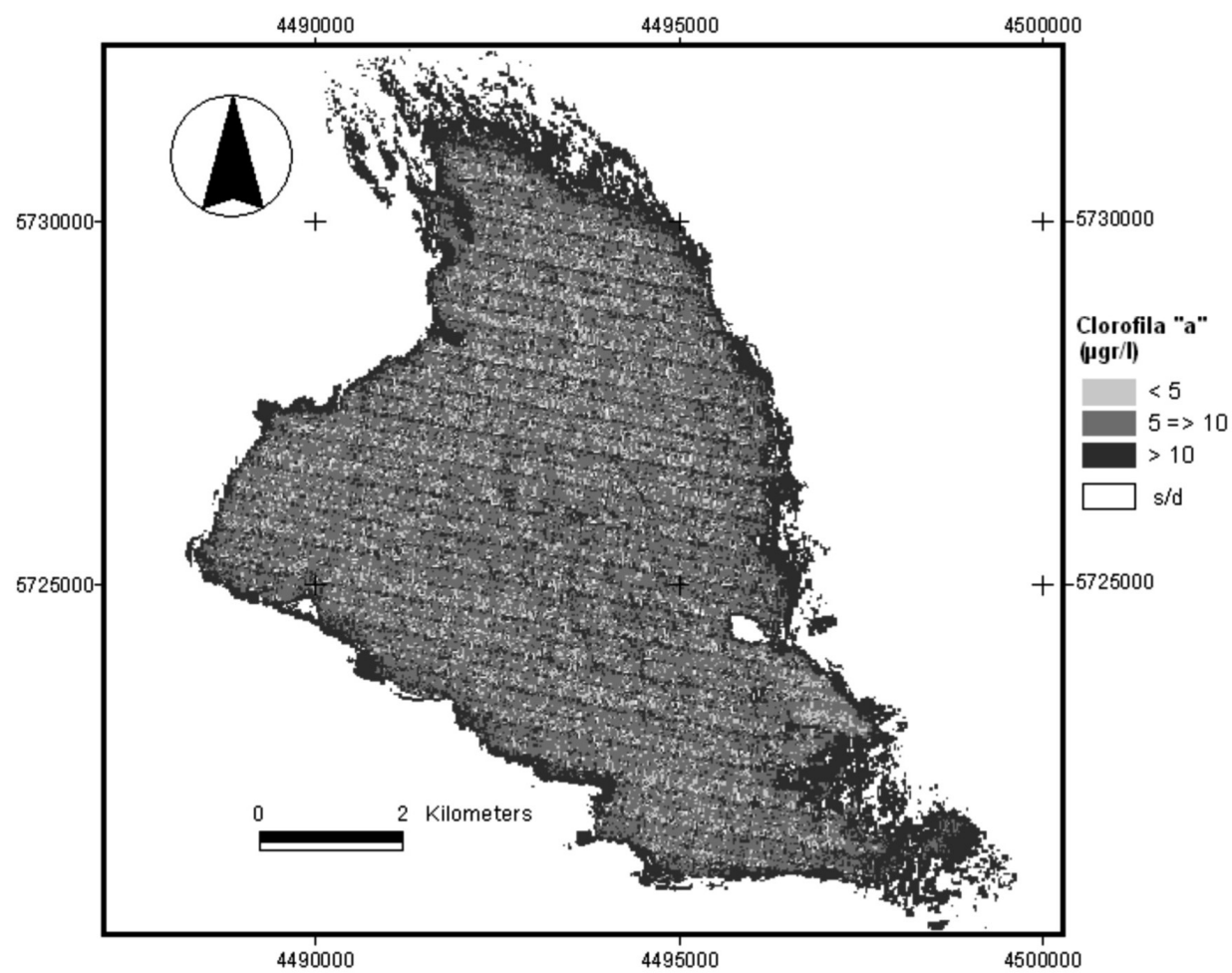

Fuente: elaboración propia.

La laguna Chasicó presentó concentraciones de clorofila «a» propias de un ambiente mesotrófico durante el período estudiado, lo cual mostró cierta homogeneidad temporal en relación a este parámetro. La utilización del NDVI como estimador de la clorofila «a» en el modelado de su distribución espacial y temporal fue exitosa. La misma mostró los mayores valores en cercanías a la costa. Dado que el método incluyó la correlación entre las concentraciones de clorofila «a» en relación a los valores de NDVI hallados en cada estación de medición, el modelado se realizó a partir de información satelital 
(píxel a píxel). Este último aspecto, presentó ventajas de precisión con respecto a una interpolación estándar de los valores hallados en cada estación de medición.

La utilización integrada de sensores de campo e imágenes satelitales mostró la importancia del monitoreo de múltiples variables y la correlación entre las mismas en el estudio de cuerpos de agua. La gran ventaja que presenta la aplicación de imágenes satelitales es la posibilidad de realizar estudios multitemporales con un área extensa de cobertura y bajos costos en relación a relevamientos de campo y estudios de laboratorio. Sin embargo, se hace énfasis en la inclusión de medición de parámetros in situ y análisis de muestras de agua en la etapa de validación de investigaciones de esta índole. La misma debe ser considerada relevante. La aplicación de este método permitirá el conocimiento acerca del estado trófico de la laguna en períodos anteriores o bien para el monitoreo periódico en el futuro.

\section{Agradecimientos}

La presente investigación fue realizada mediante subsidios otorgados por el Consejo Nacional de Investigaciones Científicas y Técnicas (CONICET) y la Universidad Nacional del Sur (UNS) (Proyecto PGI «Estudio Integrado de lagunas pampeanas, provincia de Buenos Aires»). Los autores agradecen a la Comisión Nacional de Actividades Espaciales (CONAE), al U.S. Geological Survey (USGS), al Departamento de Geografía y Turismo (UNS), al personal técnico y de laboratorio del Instituto Argentino de Oceanografía (IADO-CONICET) y al Sr. Ariel Rule (por su colaboración durante el trabajo de campo).

Fecha recepción: 13/07/2012

Fecha de aceptación: 12/12/2012

\section{BiBLIOGRAFÍA}

Bohn, V. Y., Piccolo, M. C. y Perillo, G. M. E. (2007): "Potencial uso agrícola del agua de la laguna Unamuno. Buenos Aires, Argentina”. ECOSISTEMAS. Revista Científica y Técnica de Ecología y Medio Ambiente. Asociación Española de Ecología Terrestre, XVI/2, Mayo - Agosto.

Bohn, V. Y.: "Geomorfología e hidrografía de las lagunas del sur de la provincia de Buenos Aires". Tesis Doctoral. Departamento de Geografía y Turismo, Universidad Nacional del Sur, Bahía Blanca (Argentina), 2009, 233 pp. 
Bohn, V. Y., Piccolo, M. C., Perillo, G. M. E., Pratolongo, P. D. y Fornerón, C. F. (2012): "Caracterización óptica de las lagunas Sauce Grande y Chasicó (provincia de Buenos Aires)". Biología Acuática, 27, pp. 51-60.

Bonorino, A. G. (1991): "Evaluación de la recarga de las Aguas Subterráneas en el Área de la Vertiente Occidental de las Sierras Australes". Revista Asociación Geológica Argentina, XLVI/1-2, pp. 93-102.

Campo, A., Capelli, A. y Diez, P. (2004): El clima del Sudoeste Bonaerense. Bahía Blanca, Departamento de Geografía y Turismo, Universidad Nacional del Sur, 99 pp.

Canziani, G., Ferrati, R., Marinelli, C. y Dukatz, F. (2008): "Artificial neural Networks and remote sensing in the análisis of the highly variable pampean shallow lakes". Mathematical Biosciences and Engineering, 5/4, pp. 691-711.

Carlson, R. (1977): “A Trophic State Index for Lakes". Limnology and Oceanography, 22/2, pp. 361-369.

Chuvieco Salinero, E. (2002): Teledetección Ambiental: La observación de la Tierra desde el Espacio. Barcelona, Ariel Ciencia, 586 pp.

Dangavs, N. (2005): "Los ambientes acuáticos de la provincia de Buenos Aires", en Relatorio del XVI Congreso Geológico Argentino. Geología y Recursos Minerales de la provincia de Buenos Aires, La Plata, Capítulo XIII, pp. 219-235.

Ferrati, R., Cepeda, R., Marinelli, C., Dukatz, F. y Canziani, G. (2008): "Espiando lagunas con satélites. Los colores delatan su estructura", en F. Grosman (comp.): Espejos en la Llanura. Nuestras lagunas de la Región Pampeana. Buenos Aires, UNICEN, pp. 130-138.

Ferrelli, F., Bohn, V. Y. y Piccolo, M. C. (2012): "Variabilidad de la precipitación y ocurrencia de eventos secos en el sur de la provincia de Buenos Aires", en Universidad Nacional del Sur (ed.): IX Jornadas Nacionales de Geografía Física. Buenos Aires, Departamento de Geografía y Turismo, pp. 15- 28.

Fiorentino, C. E. y Paoloni, J. D. (2001): "Evaluación hidrológica de la laguna Chasicó como base de una adecuada gestión en la explotación del recurso", en Gobierno de Chile e Instituto Interamericano de Cooperación para la Agricultura. Agua, Vida y Desarrollo (eds.): III Encuentro de las Aguas, Santiago de Chile, 24-26 oct. 2001, pp. 1-12.

Garibotti, E. J., Cervellini, P. M. y Piccolo, M. C. (2009): "Microcrustáceos planctónicos y características limnológicas de dos lagunas pampeanas (Buenos Aires, Argentina)". Limnetica, 28/1, pp. 91-104.

Glave, A. (2006): "Influencia climática en el sudoeste bonaerense y sudeste de La Pampa". Acaecer, 31/360, pp. 18-23.

Grosman, F. y Sanzano, P. (2008): "Lagunas seleccionadas y rutina de muestreo", en F. Grosman (comp.): Espejos en la Llanura. Nuestras lagunas de la Región Pampeana. Buenos Aires, UNICEN, pp. 39-46.

Han, L. (1997): "Spectral Reflectance with Varying Suspended Sediment Concentrations in Clear and Algae-Laden Waters". Photogrammetric Engineering E Remote Sensing, 63/6, pp. 701-705. 
Hudson, S. J., Moore, G. F., Bale, A. J., Dywer, K. R. y Aitken, J. (1994): “An operational approach to determining suspended sediment distributions in the Humber estuary by airborne multispectral imagery", en Environmental Research Institute of Michigan (ed.): Proceedings of the First Airborne Remote Sensing Conference and Exhibition, Strasburg (France), 12-15 September 1994. Michigan, University of Minnesota.

Hutchinson, G. E. (1957): A treatise on limnology, I. New York, J. Wiley, 660 pp.

Jensen, J. R. (2000): Remote Sensing of the Environment: An Earth perspective. New Jersey, Prentice Hall.

Kopprio, G. A., Freije, R. H., Strüssmann, C. A., Kattner, G., Hoffmeyer, M. S., Popovich, C. A. y Lara, R. J. (2010): "Vulnerability of pejerrey Odontesthes bonariensis populations to climate change in pampean lakes of Argentina". J Fish Biol, 77/8, pp. 1856-1866.

Lindell, T., Pierson, D., Premazzi, G. y Zilioli, E. (1999): Manual form monitoring European lakes using remote sensing techniques. Luxemburgo, Official Publications of the European Communities, $161 \mathrm{pp}$.

Mather, P. M. (2004): Computer Processing of Remotely-Sensed Images. Inglaterra, John Wiley \& Sons Ltd., 319 pp.

Ocean Optics (2001-2007): USB2000+ Fiber Optic Spectrometer Installation and Operation Manual. Document Number 270-00000-000-02-0807. Halma Group Company.

Pinilla Ruiz, C. (1995): Elementos de Teledetección. Madrid, Ra-ma, 344 pp.

Remes Lenicov, M. y Berasain, G. (2004): "Laguna Chasicó, Partido de Villarino. Campaña de relevamientos limnológicos e ictiológicos. Informe técnico No 66 ". Buenos Aires, Dirección Desarrollo Pesquero, Subsecretaría de actividades pesqueras, $24 \mathrm{pp}$.

Ringuelet, R. A. (1972): "Ecología y biocenología del hábitat lagunar o lago del tercer orden de la región neotrópica templada (Pampasia Sudoriental de la Argentina)". Physis, 31/82, pp. 55-76.

Rodríguez, C., Bonansea, M., Bonatto, F., Reynoso, V., Prosperi, C., Mancini, M., y Ledesma, C. (2007): "Remote Sensing -GIS to Predict on the Risk of Eutrophication in Aquatic Systems", en ONU (ed.): Space Technology for E-health. Space technology-based tele-health project initiatives in Latin America and the Caribbean. United Nations Programme on Space Applications. New York, United Nations.

Sepulcri, M. G., Pizarro, M. J., Flamenco, E., Herrera, M., Borus, J. y Giordano, L. (2012): "Cartografía de susceptibilidad hídrica en el delta del río Paraná". Revista de Investigaciones Agropecuarias (RIA), 38/2.

Song, C., Woodcock, C. E., Seto, K. C., Lenney, M. P. y Macomber, S. A. (2001): “Classification and change detection using Landsat TM data: when and how to correct atmospheric effects?". Remote Sensing of Environment, 75, pp. 230-244.

Sosnovsky, A. y Quirós, R. (2005): "Efectos de la intensidad de uso de la tierra en pequeñas lagunas pampeanas (Argentina)", en I. Vila y J. Pizarro (eds.): Tercer Taller Internacional de Eutrofización de Lagos y Embalses. Santiago de Chile, Patagonia Impresores, 197-215 pp. 
Sváb, E., Tyler, A. N., Preston, T., Présing, M. y Balogh, K. V. (2007): "Characterizing the spectral reflectance of algae in lake waters with high suspended sediment concentrations". International Journal of Remote Sensing, 26, pp. 919-928.

Thiemann S. y Kaufmann, H. (2000): "Determination of chlorophyll content and trophic state of lakes using field spectrometer and IRS-1C satellite data in the Mecklenburg lake district, Germany". Remote Sensing of Environment, 73, pp. 227 235.

\section{RESUMEN}

El objetivo de este trabajo fue evaluar la correlación entre sensores de campo e imágenes satelitales en la caracterización bio-óptica de la laguna Chasicó (Argentina) en el período febrero-abril de 2010. Los parámetros morfométricos del cuerpo de agua se obtuvieron a partir de mediciones in situ y datos satelitales. Los sensores empleados permitieron la adquisición de firmas espectrales en las que se detectaron algunas características del agua superficial: turbidez, clorofila «a» y variaciones de profundidad. Como resultado del análisis morfométrico, se obtuvo que la laguna superó los 50 km² de extensión y presentó una forma subrectangular elongada. A partir del procesamiento de imágenes satelitales, datos de clorofila «a» y el cálculo del Índice de Vegetación de Diferencia Normalizada (en inglés, NDVI), se modeló la distribución espacial del pigmento en la laguna. El 53,2\% de su área presentó entre 5 y 10 g/l de clorofila «a», por lo que fue caracterizada como un lago mesotrófico para el período estudiado.

PALABRAS CLAVE: firma espectral; clorofila «a»; parámetros morfométricos; imágenes satelitales; NDVI; mediciones in situ.

\section{Abstract}

The aim of this study was to evaluate the correlation between field sensors and satellite images in the bio-optical characterization of Lake Chasicó (Argentina) during the February-April 2010 period. The morphometric parameters of the body of water were obtained from in situ measurements and satellite data processing. The sensors that were used allowed spectral signatures to be acquired and then surface water characteristics were detected: turbidity, chlorophyll-a and depth variations. Results obtained from the morphometric analysis included that the lake surpassed the $50 \mathrm{~km}^{2}$ extension and showed a sub-rectangular elongated shape. The chlorophyll-a spatial distribution in the lake was modeled from the satellite image processing, in situ chlorophyll-a data and the Normalized Difference Vegetation Index (NDVI) calculation. $53.2 \%$ of its area showed a chlorophyll-a range between 5 and $10 \mathrm{~g} / \mathrm{l}$. Therefore, the body of water was characterized as a mesotrophic lake, during the studied period.

KEY WORDS: spectral signature; chlorophyll-a; morphometric parameters; satellite image; NDVI; in situ measurements. 
RESUMÉ

Le but de cette étude était d'évaluer la corrélation entre les capteurs de champ et l'imagerie satellitaire dans la caractérisation bio-optique de la lagune Chasicó (Argentine) pendant la période de février à avril 2010. Les paramètres morphométriques de la masse d'eau ont été obtenus à partir des mesures de données obtenues in situ et satellitaires. Les capteurs utilisés ont permis d'acquérir des signatures spectrales qui ont été détectées dans certaines caractéristiques de l'eau de surface : la turbidité, la chlorophylle «a» et les variations de profondeur. Comme résultat de l'analyse morphométrique, nous avons constaté que la superficie de la lagune dépassait les $50 \mathrm{~km}^{2}$ et qu'elle présentait une forme allongée subrectangulaire. À partir du traitement de l'image satellite, des données de chlorophylle «a» et du calcul de l'Indice de Végétation de la Différence Normalisée (en anglais, NDVI), nous avons modélisé la distribution spatiale du pigment dans la lagune. 53,2\% de la zone présente entre 5 et $10 \mathrm{~g} / \mathrm{l}$ de chlorophylle «a», ce qui permet de la cataloguer comme un lac mésotrophe pour la période étudiée.

MoTS CLES: signature spectrale; chlorophylle «a»; paramètres morphométriques; imagerie satellitaire; NDVI; mesures in situ. 